\title{
Design and Characterization of a Microreactor for Monodisperse Catalytic Droplet Generation at both Elevated Temperatures and Pressures
}

\author{
J.C. Vollenbroek, J.G. Bomer, A. van den Berg, M. Odijk \\ BIOS Lab-on-a-Chip Group, MESA+ Nanolab cleanroom \\ MESA+ Institute for Nanotechnology, University of Twente \\ Enschede, The Netherlands
}

\author{
A.E. Nieuwelink, B.M. Weckhuysen \\ Inorganic Chemistry and Catalysis \\ Debye Institute of Nanomaterials Science, Utrecht University \\ Utrecht, The Netherlands \\ R.G. Geitenbeek, A. Meijerink \\ Condensed Matter and Interfaces \\ Debye Institute of Nanomaterials Science, Utrecht University \\ Utrecht, The Netherlands \\ R.M. Tiggelaar \\ MESA+ NanoLab cleanroom \\ MESA+ Institute for Nanotechnology, University of Twente \\ Enschede, The Netherlands
}

\begin{abstract}
We report the fabrication and characterization of a microfluidic droplet microreactor with potential use for single catalyst particle diagnostics. The aim is to capture Fluid Catalytic Cracking (FCC) particles in droplets and perform a probe reaction that results in a fluorescent output signal. The intensity of such a signal can be used as a measure of the catalytic activity of the particle. The microreactor features a droplet generator, platinum (Pt) microheaters, and Pt micro temperature sensors, and is able to operate at pressures up to at least 5 bar. Fluidic channels are etched in a silicon substrate, and platinum heater and sensor structures embedded in the glass cover. We have mapped the temperature inside the microchannels using nanoparticles that show temperature-dependent luminescence. At various spots on the chip, the temperature deviates by $\mathbf{0 . 8 6}$ degrees Celsius close to the Pt sensor and 5.5 degrees Celsius farther away from it. Experiments with making oil-in-water droplets at various temperatures and pressures result in stable droplets up to $\mathbf{1 0 0}$ degrees Celsius at atmospheric pressure. At this temperature, small gas bubbles are formed in the water phase, and then collected by the oil droplets. At a pressure of 5 bar, the droplets are stable up to at least 120 degrees Celsius. E-cat FCC particles were captured in water droplets at a rate of 150 droplets per second.
\end{abstract}

Keywords-microreactor; droplet microfluidics; E-cat FCC; Pt microheater; elevated temperature and pressure

This work was supported by the Netherlands Center for Multiscale Catalytic Energy Conversion (MCEC), an NWO Gravitation programme funded by the Ministry of Education, Culture and Science of the government of the Netherlands.

\section{INTRODUCTION}

Fluid Catalytic Cracking (FCC) is an essential process in the production of gasoline. The heterogeneous catalyst that is used in this process consists of several components of which zeolite crystals are the main active component [1]-[3]. Over the long term, catalyst particles deactivate during the harsh FCC process and fresh catalyst material is fed into the reactor constantly to maintain its overall activity. Metal poisoning and coke formation are examples of contamination that reduce the efficacy of an FCC particle [1]. Characterization of the spent particles is currently done in bulk, from which only ensemble averages are obtained. However, due to the large age distribution in spent catalyst material, they are very heterogeneous in nature. Therefore, there is need for a fast screening of single particles to give more insight in this inter-particle heterogeneity. In this paper we present a microfluidic platform dedicated to single catalyst particle diagnostics, consisting of a droplet generator, capable of generating up to 150 droplets per second, and a microheater. Inside the droplets, the activity of a single particle can be studied using fluorescence microscopy.

\section{A. Catalytic activity}

As the catalytic activity of FCC particles is linked to the availability of acid sites in their zeolite domains, the activity of 
FCC particles can be studied with a probe molecule like styrene. The oligomerization of styrene is acid catalyzed and occurs at temperatures between 100 and $200{ }^{\circ} \mathrm{C}$. The oligomerization products are fluorescent and can therefore be characterized with UV-vis [2] and fluorescence microscopy [4]. These two microspectroscopic techniques are combined in [3] to investigate the acidity of different FCC particles with styrene-like probe molecules.

\section{B. The use of droplets in microfluidics}

In the field of microfluidics, the use of microdroplets is common practice. Microdroplets are used in many applications such as chemical microreactors, single particle traps and biological assays of cells and DNA [5]-[8]. By mixing two immiscible fluids such as oily (nonpolar) and watery (polar) substances, either oil-in-water $(\mathrm{O} / \mathrm{W})$ or water-in-oil $(\mathrm{W} / \mathrm{O})$ droplets are created [5], [6], [9]. A droplet is the result of the shear forces and interfacial tension between the two fluids, creating a stream of liquid A (continuous phase) with droplet of liquid $B$ (dispersed phase) [6]. It is vital that the walls of the channel have a preference for wetting by the opposite phase than that of the droplet, as demonstrated in [9]. Droplets form an ideal reaction environment because of their well-controlled properties such as shape, size, and monodispersity, creating a homogeneous reaction environment. The configuration of the channels that are used for the creation of droplets also plays a role. Examples of geometry types are the Capillary, the Tjunction, and the Flow Focusing junction [6].

\section{Microheaters}

To obtain the temperature range in which reactions that require elevated temperatures can occur, thin film platinum structures that can be heated via joule heating are incorporated on the chip. These platinum heaters are stable up to at least $500{ }^{\circ} \mathrm{C}$ [10], [11], depending on the adhesion layer used. These thin film microheaters have been widely reported in the literature [10]-[21], and vary from use in biological assays [15]-[17], [19], [20], to reactors or hotplates for single phase purposes [11]-[13], [18], and finally to reactions inside droplets on a microfluidic chip [16], [22]. In these last two cases, this was either achieved in stationary droplets [16], or using bulky heating systems [22]. The ability to use microfluidic systems at elevated pressures has been demonstrated previously [23], [24], where combinations of both high pressure and high temperature are reported in [23].

Although heterogeneous catalysis has been performed before in droplets on a millifluidic chip [22], this has not yet been achieved using an integrated heater section, but in an oven. Other work on microreactors either did not involve droplets [11]-[13], [18], or if it did, they were in bulky systems [22], and involved stationary droplets [16]. Our single catalyst particle diagnostic platform is designed to measure the activity of FCC particles and offers a well-controlled reaction environment with respect to both volume and temperature; all features integrated into a single microfluidic chip, and without bulky control systems. The throughput is - in theory - limited by both the optical detection method, and the time needed for the reaction to give a measurable fluorescence signal, for a given channel length and concentration of reactants.

\section{DESIGN}

The chip shown in Fig. 1 (A) shows the design of the microreactor. It has several features, including a droplet generator (C), a microreactor and optical window (B), and a temperature sensor section (D). Oil flows in through the main channel via inlet I2, and water through the coming from the side via inlet I1 to create droplets of oil in water. The heater section contains three separate heaters. Heater 1 and heater 2 (H1 and H2) can be controlled separately via electrodes E9 and E2. Both these heaters have an interlocked temperature sensor, so they can be monitored and controlled via LabVIEW software. These temperature sensors are shown in Fig. 1 (D). The optical window can be used for fluorescence measurements on the droplets, although the heaters are a small enough width to monitor the droplets underneath the heaters as well. A four-point measurement, in which a current is applied to electrodes E3 and E8, thereby inducing a voltage drop over the small resistor structure, is used to measure the resistance of the sensor. This voltage can be measured between electrodes E4 and E5 for S1, and electrodes E6 and E7 for S2. When the temperature increases, the resistance increases, resulting in an increasing voltage across the specified electrodes. The final heater section, H3, is a block of parallel heaters. These heaters do not have a dedicated temperature sensor. The parallel heaters are connected to E10; E1 is the ground electrode to which all heaters are connected. The parallel heater section, as well as the meandering structure of the channels, are necessary to give the reaction enough time to occur at elevated temperatures. However, this standard design has some variations. The channel width varies between $150-300 \mu \mathrm{m}$, in steps of $50 \mu \mathrm{m}$. For some chips, a Tjunction is used as the droplet generator, and finally the area that is covered by the parallel heater is varied between the various chips. The overall dimensions of the chip are $15 \mathrm{~mm} \times 20 \mathrm{~mm} \times$ $1 \mathrm{~mm}$.

\section{EXPERIMENTAL}

\section{A. Fabrication}

The chips were fabricated in the cleanroom of the MESA+ NanoLab at the University of Twente. Fig. 2 shows an overview of the process steps followed to fabricate the chip. The chip is a stack of a silicon and a glass substrate. A three-mask process is used to develop the desired features both in and on the substrates, as shown in Fig. 2. Using BHF as a wet etchant, the pattern for the Pt structures is etched $200 \mathrm{~nm}$ deep into the glass substrate. This process is directly followed by the deposition of a $10 \mathrm{~nm}$ thick tantalum adhesion layer and a $190 \mathrm{~nm}$ thick platinum layer, causing the heater to be embedded into the glass substrate. Next, the complete glass substrate containing the Pt structures is covered with $1 \mu \mathrm{m}$ PECVD $\mathrm{SiO}_{2}$. The $\mathrm{SiO}_{2}$ is then removed from the contact electrodes. The channels are etched $150 \mu \mathrm{m}$ deep into the silicon substrate using DRIE etching. Fluidic and electronic accesses are formed on the backside of the substrates are bonded together. However, before this final step, the silicon substrate is first processed in a furnace for the dry oxidation of $\mathrm{Si}$, to create a the $200 \mathrm{~nm}$ thin insulating $\mathrm{SiO}_{2}$ film on the walls of the channel. After completion of all the process 


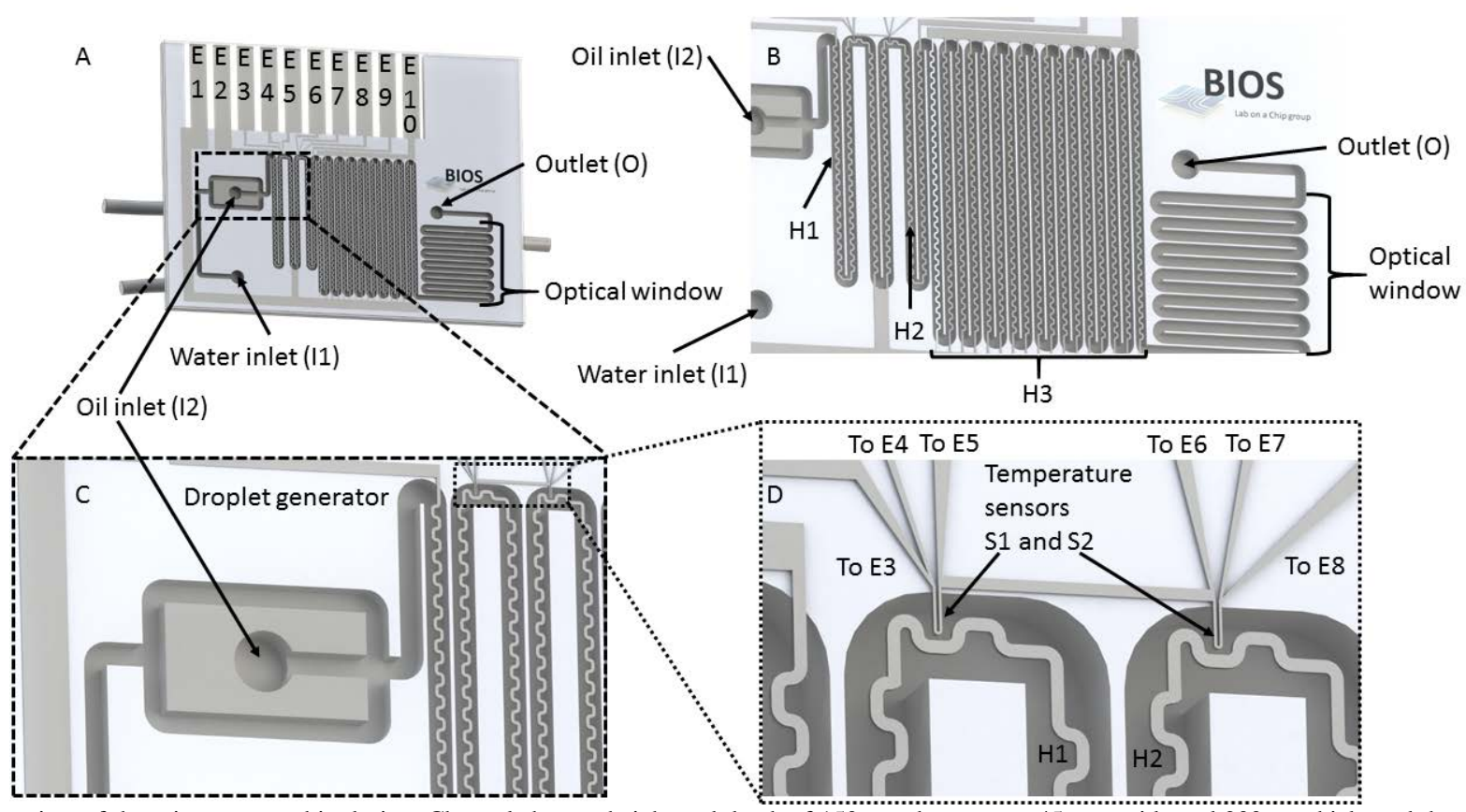

Fig. 1. Overview of the microreactor chip design. Channels have a height and depth of $150 \mu \mathrm{m}$, heaters are $45 \mu \mathrm{m}$ wide and $200 \mathrm{~nm}$ thick, and the temperature sensor has a width of $10 \mu \mathrm{m}$ and thickness of $200 \mathrm{~nm}$. All heater-related structures are made out of platinum; the bottom substrate is silicon and the top substrate is glass. Furthermore, the picture contains images of A) broad overview showing the electrodes (E1-E10), channel layout, inlets for the dispersed phase (I1) and continuous phase (I2), and the outlet (O). B) Close-up of the heater sections showing the three heaters (H1 - H3) that can be controlled via electrodes E2 (H1), E9 (H2), and E10 (H3). C) Close-up of the droplet generator feature, showing the flow focusing junction and the inlet for the dispersed phase. D) Close-up of the temperature sensor structures (S1 and S2) combined with the electrodes used for addressing those structures (E3 - E8).

steps, the silicon and glass substrates are anodically bonded, and the individual chips are created as a result of dicing.

\section{B. Pt temperature sensor characterization}

Characterization of the Pt temperature sensors is achieved by mounting the chip on a Printed Circuit Board (PCB) and wirebonding the electrodes to the $\mathrm{PCB}$, so the chip can be addressed via external connectors. The chip is fully immersed in a beaker of oil standing on an IKA RET hotplate. A thermocouple thermometer, connected to the hotplate, is used as a reference thermometer and measures the temperature of the oil. A magnetic stirring bead is added to increase the uniformity of the temperature distribution in the oil. The hotplate is cycled between $30{ }^{\circ} \mathrm{C}$ and $150{ }^{\circ} \mathrm{C}$, to rule out any hysteresis effects.

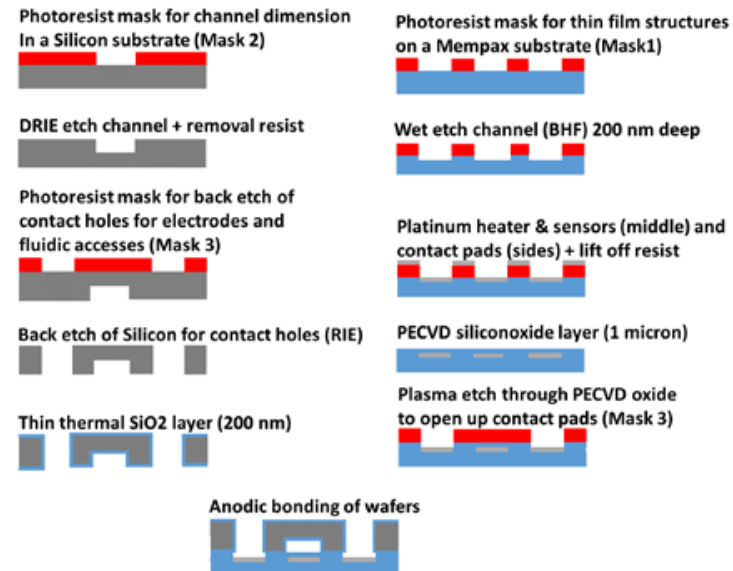

Fig. 2. Schematic cross-sectional representation of the various steps used in the process of fabrication of the droplet microreactor.
Meanwhile, the resistance of the temperature sensor on the microreactor is measured. A signal from the sensor is measured by applying a current; using a LM317TG voltage regulator as the current source. The voltage across the resistor is amplified using a AD620ANZ instrumentation amplifier. The output signal is recorded by the Nationl Instruments (NI) myRio data acquisition board, which is connected to LabVIEW software on a computer.

\section{Temperature validation using temperature dependent luminescent nanoparticles}

To validate whether the temperature measured on the outside of the channel by the Pt sensor is an accurate representation of the temperature inside the channel, $\mathrm{NaYF}_{4}: \mathrm{Er}^{3+}, \mathrm{Yb}^{3+}$ nanoparticles that show temperature-dependent luminescence are used to determine the temperature inside the microchannel. The nanoparticles were dispersed in 1-Octadecene (ODE) and flushed through the chip. The particles are excited by a 2 W 980 nm laser and through upconversion emit light with distinct peaks in both the $500-535 \mathrm{~nm}$ and $535-570 \mathrm{~nm}$ regions. Spectra are collected using an Ocean Optics QE Pro CCD camera equiped with a $500 \mathrm{~nm}$ grating. The temperature-dependent luminescence is due to a Boltzmann distribution in two energy levels with an energy difference of several $k T$. With increasing also temperature, the ratio between the height of the peak regions increases, giving a measure of the temperature. The temperature dependent luminescence was calibrated before the experiment, and Fig. 3 shows the relation between the natural logarithm of the fluorescence intensity ratio (FIR) and $1 / T$ in $K^{-1}$, which is as expected for thermally coupled states. While flushing the chip with the nanoparticle dispersion, the temperature was measured 


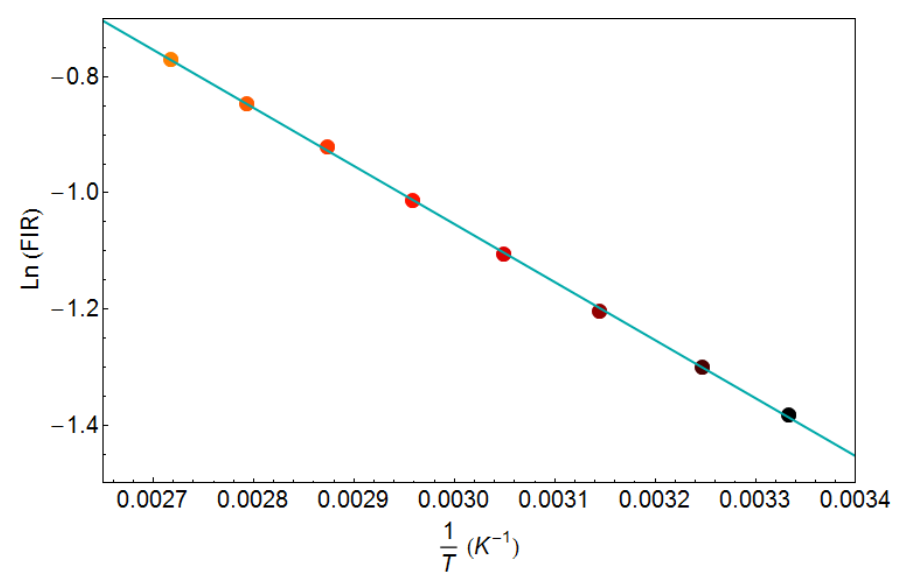

Fig. 3. Natural logarithm of the fluorescence intensity ratio (FIR) at $28^{\circ} \mathrm{C}$, $35^{\circ} \mathrm{C}, 45^{\circ} \mathrm{C}, 55^{\circ} \mathrm{C}, 65^{\circ} \mathrm{C}, 75^{\circ} \mathrm{C}, 85^{\circ} \mathrm{C}$ and $95^{\circ} \mathrm{C}$ (black to orange). The intensities are normalized on the peak at $541 \mathrm{~nm}$. The linear correlation between the $\log (\mathrm{FIR})$ and $1 / T$. The linear fit, $\operatorname{Ln}(\mathrm{FIR})=1.97814-1010.99 / T$, is of high quality with an $\mathrm{R}^{2}$ of 0.999 .

at two spots on the chip. The first spot was close to sensor S1 and the second spot was $8 \mathrm{~mm}$ to the right of spot 1 , near the optical window. During these measurements, only $\mathrm{H} 1$ and $\mathrm{H} 2$ were activated. The temperature was cycled between $70^{\circ} \mathrm{C}$ and $140{ }^{\circ} \mathrm{C}$.

\section{Droplet creation}

Droplets are created by connecting a Nemesys syringe pump, fitted with Hamilton syringes of $500 \mu L$ (oil) and $1000 \mu L$ (water), and fused silica tubing (Polymicro Technologies, ID = $250 \mu \mathrm{m}, \mathrm{OD}=360 \mu \mathrm{m}$ ) to the oil and water inlets of the microreactor. The pump is controlled by Nemesys software. For the dispersed phase FC-40 oil is used and for the continuous phase MilliQ demiwater. A Photron SA3 high-speed camera and FPV software, in combination with a Leica DMi 5000 M microscope, are used to capture movies and images from the droplets as they are created. Experiments at high pressure are performed by adding a P786 backpressure regulator from Idexhs, at a backing pressure of 5 bar. For the creation of water in oil droplets the channel walls were made hydrophobic by flushing the chip with a solution of $5.55 \mu L$ tridecafluoro-1,1,2,2tetrahydrooctyltrichlorosilane (FOTS) added to $1.5 \mathrm{~mL}$ FC-40 oil. The chip is flushed for 5 minutes with the FOTS and FC-40 solution and then for 5 minutes with only FC-40. E-Cat FCC particles were added to the syringe containing the dispersed phase in order to capture the particles in droplets.

\section{RESUltS}

\section{A. Temperature sensor characterization and heater control}

Temperature characterization was performed using the oil immersion method as described in the experimental section. The Pt sensors show a sensitivity $0.0476 \Omega^{\circ} C^{-1}$, and exhibit a high linearity, given the $\mathrm{R}^{2}$ of 0.99899 for $\mathrm{S} 1$ and an $\mathrm{R}^{2}$ of 0.9989 for S2. Offsets are $18.311 \Omega$ and $18.294 \Omega$ for S1 and S2 respectively. Sensitivity and offset values are used describe the linear relation between the resistance ( $\mathrm{R}$ in $\Omega$ ), and temperature ( $\mathrm{T}$ in ${ }^{\circ} \mathrm{C}$ ), of the $\mathrm{Pt}$ sensors: $R=0.0476 T+18.311$. The resolution of the temperature sensors is $0.15^{\circ} \mathrm{C}$, determined by the 12-bit ADC of the myRio and the amplification factor of the readout circuit. Testing the control of the heater was done by varying the setpoint of a PI control system in LabVIEW on the NI myRio. The setpoint of the control loop was varied between $23^{\circ} \mathrm{C}$ and $120^{\circ} \mathrm{C}$; during these experiments droplets were created at the same time inside the microreactor. The step response time (the time needed to reach the setpoint) is approximately 1.5 seconds after which the temperature oscillates around the setpoint. There is a standard deviation of $2.6{ }^{\circ} \mathrm{C}$ around the setpoint. The parallel heaters (H3) can be manually controlled using the software. They are connected to a different NMOS than $\mathrm{H} 1$ and $\mathrm{H} 2$. The parallel heaters generate enough heat to be measured by S1 and S2. By carefully adjusting the duty cycle of H3, it can be optimally tuned for assisting H1 and H2 in uniformly heating .

\section{B. Temperature validation using nanoparticles}

Fig. 4 shows the difference in temperature reading, in the range of $70^{\circ} \mathrm{C}$ to $140{ }^{\circ} \mathrm{C}$, between the temperature-dependent luminesence and $\mathrm{Pt}$ sensor $\mathrm{S} 1$, where (A) shows a maxium difference of $0.86{ }^{\circ} \mathrm{C}$ at spot 1 close to sensor S1 and (B) shows a maximum difference of $5.5^{\circ} \mathrm{C}$ at spot $2(8 \mathrm{~mm}$ to the right of spot 1$)$. Note that during these experiments only $\mathrm{H} 1$ and $\mathrm{H} 2$ were activated. The calibration curve shown in Fig. 3 was used to convert the measured luminescence intensities to temperatures.

\section{Droplets at various temperatures and pressures}

Droplets were created using the setup described in section D of the experimental procedure. Several tests were performed on droplets being created under various conditions, as shown in Fig. 5. In (A) the heaters are switched off, in (B) the temperature is kept at $50{ }^{\circ} \mathrm{C}$ and in (C) $100{ }^{\circ} \mathrm{C}$, in (D) the temperature is above the boiling point of water $\left(120^{\circ} \mathrm{C}\right)$, and finally in (E) the temperature is above the boiling point of water $\left(120^{\circ} \mathrm{C}\right)$, but the system is at a pressure of 5 bar. For all measurements, the flow rates of the water and oil phase are $15 \mu \mathrm{L} / \mathrm{min}$ and $3 \mu \mathrm{L} / \mathrm{min}$ respectively, and images were taken using the high-speed camera at a framerate of $125 \mathrm{fps}$. The width of the channel for these experiments was $250 \mu \mathrm{m}$. Fig. 5. (A) shows an image of droplets created at room temperature. The created droplets are highly monodisperse, as shown in Fig. 5. (B), in which droplets at $50{ }^{\circ} \mathrm{C}$ are shown. The volume of the droplet is approximately $47 \mathrm{~nL}$ and does not change with temperature. When the temperature is increased to $100{ }^{\circ} \mathrm{C}$, small gas bubbles start to appear in the channel, as is shown in Fig. 5. (C). The bubbles find their origin under the heater and when they encounter an oil droplet they show affinity towards the oil phase. Inside the oil bubble the small bubbles seem to follow the path of the heater. At $120^{\circ} \mathrm{C}$, large gas bubbles are formed and the droplets start to break up, showing a dewetting phenomenon at the channel ceiling surface. A collapsed droplet, with large gas bubbles in the droplet, is shown in Fig. 5 (D). Once the backpressure regulator is added, the droplets are still intact at $120^{\circ} \mathrm{C}$. This is shown in Fig. 5 (E). The monodispersity is maintained when working above atmospheric pressure at the outlet. 
A

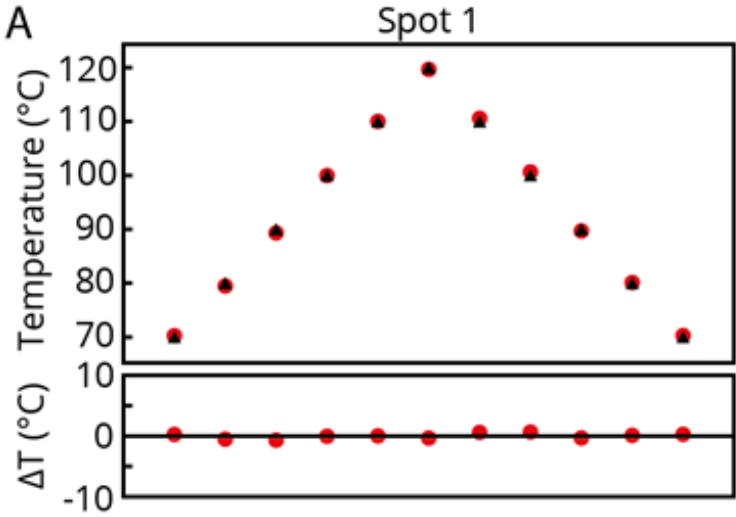

B

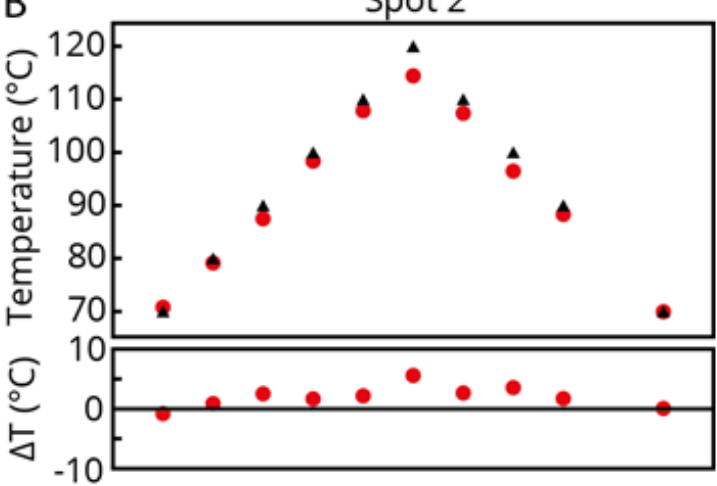

$\Delta$ Temperature indicated by Pt sensor

\section{- Temperature indicated by luminescent} nanoparticles

Fig. 4. Temperatures determined by the Pt sensors S1 (black triangles) and luminescent nanoparticles (red circles) at (A) spot 1 and (B) spot 2. The difference between the two measured values is shown at the bottom of the pictures.

\section{FCC in water droplets}

Experiments showed that the FCC particles had a high affinity for the $\mathrm{SiO}_{2}$ wall of the channel and preferred to stick there instead of staying suspended in the flow. Furthermore, they preferred the water phase to the oil phase. The channels and tubing were made hydrophobic by coating with FOTS, as described in the experimental section. Fig. 6 shows FCC particles captured in water droplets. Flowrates of $60 \mu \mathrm{L} / \mathrm{min}$ for the water phase and $120 \mu \mathrm{L} / \mathrm{min}$ for the oil phase are used. Applying these flowrates, up to 150 droplets per second are generated. It should be noted that the capture efficiency of one particle per droplet is low, with many empty droplets. Agitating the syringe and tubing results in particle clusters yielding multiple particles per droplet. The image is captured using the high-speed camera set to 1000 fps.

\section{CONCLUSION AND RECOMMENDATION}

We fabricated a microreactor for a study on the catalytic activity of single catalyst particles inside droplets. Successful creation of droplets, as well as heating the droplets at pressures

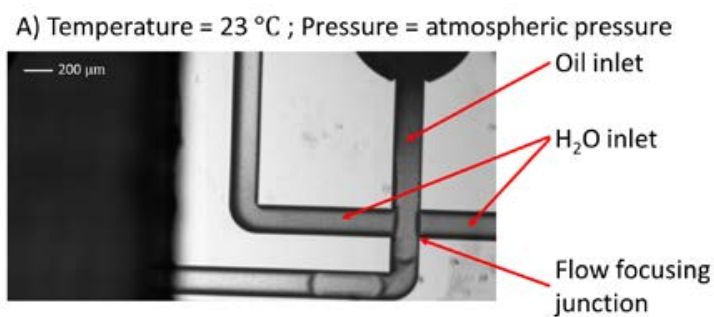

B) Temperature $=50^{\circ} \mathrm{C}$; Pressure $=$ atmospheric pressure

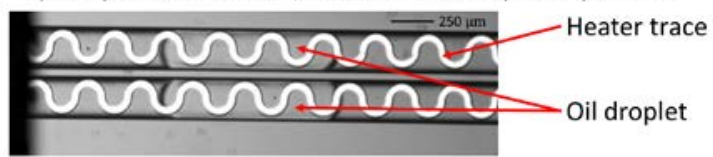

C) Temperature $=100^{\circ} \mathrm{C}$; Pressure $=$ atmospheric pressure

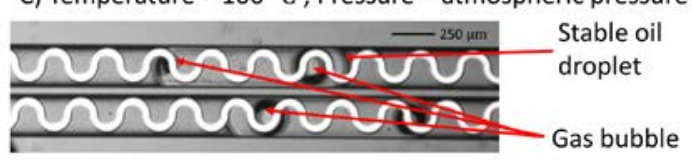

D) Temperature $=120^{\circ} \mathrm{C}$; Pressure $=$ atmospheric pressure

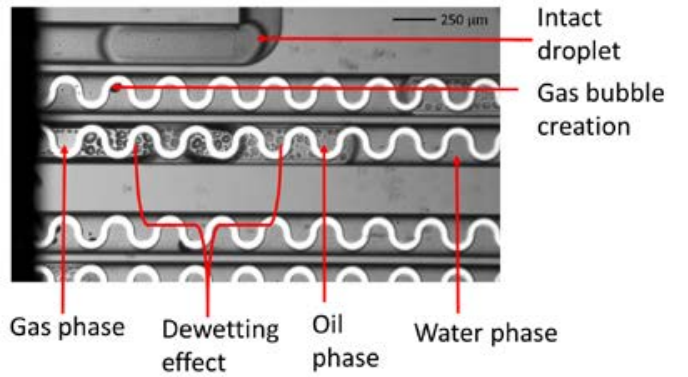

E) Temperature $=120^{\circ} \mathrm{C}$; Pressure $=5$ bar

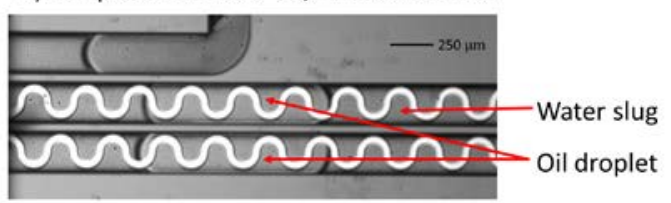

Fig. 5. Droplets created under various conditions, where at A) the heaters are switched off, B) the temperature is kept at $50{ }^{\circ} \mathrm{C}$, C) The temperature is $100{ }^{\circ} \mathrm{C}$ and small gassbubbles are collected by the droplets, D) the temperature is above the boiling point of water (at $120^{\circ} \mathrm{C}$ ), and finally $\mathrm{E}$ ) the temperature is above the boiling point of water (at $120^{\circ} \mathrm{C}$ ), but the system is at an outlet pressure of approximately 5 bar. The channel width is $250 \mu \mathrm{m}$.

up to 5 bar, has been achieved within the microreactor. The droplets generated are highly monodisperse, creating welldefined and uniform reaction environments. The temperature of the heater elements can be controlled at a standard deviation of approximately $2.6{ }^{\circ} \mathrm{C}$ from the setpoint. Measurement of the temperature can be achieved at a resolution of $0.15^{\circ} \mathrm{C}$. The $\mathrm{Pt}$ temperature sensors accurately represent the temperature inside the channel close to the sensor, which is confirmed by measurements using $\mathrm{NaYF}_{4}: \mathrm{Er}^{3+}, \mathrm{Yb}^{3+}$ nanoparticles, which show temperature-dependent luminescence. The measurements show a maximum deviation in temperature reading close to the Pt sensor of $0.86{ }^{\circ} \mathrm{C}$. Nonuniform heating results in a temperature drop toward the outlet of the chip, which results in a maximum deviation of $5.5^{\circ} \mathrm{C}$ between the Pt sensor and the nanoparticles at this spot. However, this was in an experimental 


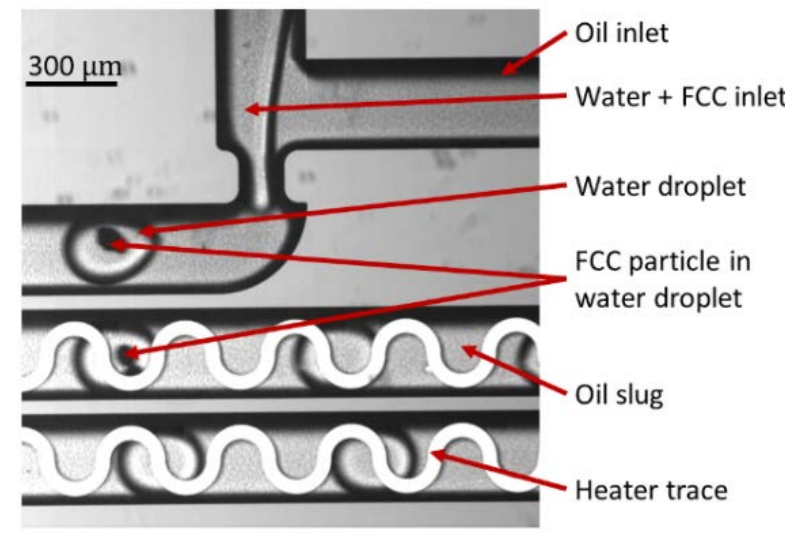

Fig. 6. E-cat FCC particles in water droplets at flow rates of $60 \mu \mathrm{L} / \mathrm{min}$ for the water phase and $150 \mu \mathrm{L} / \mathrm{min}$ for the oil phase, generating 150 droplets per second.

case with $\mathrm{H} 3$ switched off. In practice H3 is also used, thereby reducing the nonuniform distribution. Furthermore, we succeeded in capturing FCC particles in water droplets. Although the capture efficiency remains low, particles can be captured with a frequency of 150 droplets per second. Future work will concentrate on executing a suitable probe reaction catalyzed by the FCC particle. Having transferred the particles from the apolar phase to a polar phase means that the probe reaction with styrene is not suitable, since styrene is dissolved in an apolar liquid. The use of furfurylalcohol as a probe that is solvable in the polar phase will be investigated, although such a reaction does not necessarily require an elevated temperature. Furthermore, the capturing efficiency of particles requires improvement, since currently droplets containing a single particle remain scarce. In fact, the droplets are either empty or contain multiple particles. However, the use of the microreactor is not limited to solely testing FCC particles and can also be used to study the activity of other synthesized single catalyst particles.

\section{ACKNOWLEDGMENT}

The authors are grateful to S. Dekker, H. de Boer, and C. Bruinink for their valuable help with the fabrication of both chips and chipholder.

\section{REFERENCES}

E. T. C. Vogt and B. M. Weckhuysen, "Fluid catalytic cracking: recent developments on the grand old lady of zeolite catalysis," Chem. Soc. Rev., vol. 44, no. 20, pp. 7342-7370, 2015.

[2] I. L. C. Buurmans, J. Ruiz-Martínez, W. V Knowles, D. van der Beek, J. A. Bergwerff, E. T. C. Vogt, B. M. Weckhuysen, "Catalytic activity in individual cracking catalyst particles imaged throughout different life stages by selective staining.," Nat. Chem., vol. 3, no. 11, pp. 8627, 2011.

[3] L. R. Aramburo, S. Wirick, P. S. Miedema, I. L. C. Buurmans, F. M. F. de Groot, and B. M. Weckhuysen, "Styrene oligomerization as a molecular probe reaction for Brønsted acidity at the nanoscale," Phys. Chem. Chem. Phys., vol. 14, no. 19, p. 6967, 2012.

[4] E. Stavitski, M. H. F. Kox, and B. M. Weckhuysen, "Revealing shape selectivity and catalytic activity trends within the pores of H-ZSM-5 crystals by time- and space-resolved optical and fluorescence microspectroscopy," Chem. - A Eur. J., vol. 13, no. 25, pp. 70577065, 2007.

[5] S.-Y. Teh, R. Lin, L.-H. Hung, and A. P. Lee, "Droplet microfluidics.,” Lab Chip, vol. 8, no. 2, pp. 198-220, 2008.

[6] X. Casadevall i Solvas and A. J. deMello "Droplet microfluidics: recent developments and future applications.," Chem. Commun. (Camb)., vol. 47, no. 7, pp. 1936-42, 2011.

[7] J. H. Leamon, D. R. Link, M. Egholm, and J. M. Rothberg, "Overview: methods and applications for droplet compartmentalization of biology.," Nat. Methods, vol. 3, no. 7, pp. 541-543, 2006

[8] R. M. Schoeman, Droplet Microfluidic Platform for Cell Electrofusion. 2015

[9] L. Shui, A. van den Berg, and J. C. T. Eijkel, "Interfacial tension controlled W/O and O/W 2-phase flows in microchannel.," Lab Chip, vol. 9, no. 6, pp. 795-801, 2009

[10] R. M. Tiggelaar, R. G. P. Sanders, A. W. Groenland, and J. G. E. Gardeniers, "Stability of thin platinum films implemented in hightemperature microdevices," Sensors Actuators, A Phys., vol. 152, no. 1, pp. 39-47, 2009

[11] W.-Y. Chang and Y.-S. Hsihe, "Multilayer microheater based on glass substrate using MEMS technology,” Microelectron. Eng., vol. 149, pp. 25-30, 2016

[12] R. M. Tiggelaar, P. Van Male, J. W. Berenschot, J. G. E. Gardeniers, R. E. Oosterbroek, M. H. J. M. De Croon, J. C. Schouten, A. Van Den Berg, and M. C. Elwenspoek, "Fabrication of a high-temperature microreactor with integrated heater and sensor patterns on an ultrathin silicon membrane," Sensors Actuators, A Phys., vol. 119, no. 1, pp. 196-205, 2005

[13] R. M. Tiggelaar, P. W. H. Loeters, P. Van Male, R. E. Oosterbroek, J. G. E. Gardeniers, M. H. J. M. De Croon, J. C. Schouten, M. C. Elwenspoek, and A. Van den Berg, "Thermal and mechanical analysis of a microreactor for high temperature catalytic gas phase reactions,” Sensors Actuators, A Phys., vol. 112, no. 2-3, pp. 267277, 2004.

[14] V. Miralles, A. Huerre, F. Malloggi, and M.-C. Jullien, A Review of Heating and Temperature Control in Microfluidic Systems: Techniques and Applications, vol. 3, no. 1. 2013.

[15] J. L. Lin, M. H. Wu, C. Y. Kuo, K. Da Lee, and Y. L. Shen, "Application of indium tin oxide (ITO)-based microheater chip with uniform thermal distribution for perfusion cell culture outside a cell incubator," Biomed. Microdevices, vol. 12, no. 3, pp. 389-398, 2010.

[16] C. Fang, D. Lee, B. Stober, G. G. Fuller, and A. Q. Shen, "Integrated microfluidic platform for instantaneous flow and localized temperature control,” RSC Adv., vol. 5, no. 104, pp. 85620-85629, 2015

[17] T. Pennell, T. Suchyna, J. Wang, J. Heo, J. D. Felske, F. Sachs, and S. Z. Hua, "Microfluidic chip to produce temperature jumps for electrophysiology,” Anal. Chem., vol. 80, no. 7, pp. 2447-2451, 2008.

[18] A. I. K. Lao, T. M. H. Lee, I. M. Hsing, and N. Y. Ip, "Precise temperature control of microfluidic chamber for gas and liquid phase reactions,” Sensors Actuators, A Phys., vol. 84, no. 1, pp. 11-17, 2000

[19] N. Crews, C. Wittwer, and B. Gale, "Continuous-flow therma gradient PCR,” Biomed. Microdevices, vol. 10, no. 2, pp. 187-195, 2008.

[20] B. L. R. Zhong, X. Pan, L. Jiang, Z. Dai, J. Qin, "Simply and reliably integrating micro heaters / sensors in a monolithic PCR-CE microfluidic genetic analysis system,” Electrophoresis, no. 30, pp. 1297-1305, 2009

[21] R. Phatthanakun, P. Deekla, W. Pummara, C. Sriphung, C. Pantong and N. Chomnawang, "Fabrication and Control of Thin-Film Aluminum Microheater and Nickel Temperature Sensor” no. 2, pp. 14-17, 2011.

[22] K. Olivon and F. Sarrazin, "Heterogeneous reaction with solid catalyst in droplet-flow millifluidic device,” Chem. Eng. J., vol. 227, pp. 97-102, 2013

[23] K. Jensen, D. Ph, and K. F. Jensen, "Temperature Microreactors for Nanostructure Synthesis,” J. Assoc. Lab. Autom., vol. 14, no. 6, pp. 367-373, 2009.

[24] S. K. Luther and A. Braeuer, "High-pressure microfluidics for the investigation into multi-phase systems using the supercritical fluid extraction of emulsions (SFEE), ” J. Supercrit. Fluids, vol. 65, pp. 7886, 2012. 